\section{The effect of calorie restricted diet on anthropometric and biochemical parameters in Sprague Dawley rats}

Muhammad Fakhruddin Irfan Sazali and Maleeka Abdullah Hilmy Postgraduate Centre, Management and Science University, Shah Alam, Malaysia

Norshafarina Shari

Faculty of Health and Life Sciences, Management and Science University, Shah Alam, Malaysia

Lilik Herawati

Faculty of Medicine, Universitas Airlangga, Surabaya, Indonesia

Nurul Izza Nordin

Industrial Biotechnology Research Centre, SIRIM Berhad, Shah Alam, Malaysia, and

Zulhabri Othman

Postgraduate Centre, Management and Science University, Shah Alam, Malaysia and International Centre for Halal Studies, Management and Science University, Shah Alam, Malaysia

\footnotetext{
Abstract

Purpose - Calorie restricted diets are known to improve health and promote healthy aging. This happens because of controlled inflammation and metabolism in the body. This study aims to evaluate the differences in anthropometric and biochemical parameters in Sprague Dawley (SD) rats because of consumption of a calorie restricted diet.

Design/methodology/approach - The study divided SD rats $(n=15)$ into 3 groups consuming high calorie intake (HCI), medium calorie intake and low calorie intake (LCI). Anthropometric parameters were determined through measurement of abdominal circumference (AC), thoracic circumference (TC), body length and body weight (BW). Biochemical parameters analyzed in this study were fasting blood glucose level and full blood lipid profile. Nutritional status was obtained based on food consumption, energy intake (EI) and food efficiency rate. Measurements were taken for a period of four weeks.

Findings - Analysis on anthropometric parameters indicates a significant difference in mean BW between HCI $(230.44 \pm 1.47 \mathrm{~g})$ and LCI $(188.54 \pm 1.50 \mathrm{~g})$. There is a significant difference in abdominal $\mathrm{TC}$ ratio $(\phi<0.001 ; \mathrm{F}=13.599)$ in the LCI group $(1.01 \pm 0.00714)$ compared to the HCI group $(1.04 \pm 0.00858)$. Post hoc for nutrition parameters indicates a significant difference in mean EI between $\mathrm{HCI}(9.71 \pm 0.006 \mathrm{~kJ})$ and $\mathrm{LCI}(3.21 \pm 0.001 \mathrm{~kJ})$. There is a significant effect $(\phi<0.0001 ; \mathrm{F}=3042872.02)$ of EI on rats in all three groups. HDL levels were significantly higher $(p<0.0001 ; \mathrm{F}=1536.89)$ in the LCI group $(68.60 \pm 0.55 \mathrm{mg} / \mathrm{dL})$ compared to the HCI group $(49.40 \pm 0.55 \mathrm{mg} / \mathrm{dL})$. The Pearson's correlation results show a strong positive correlation in EI with $\mathrm{BW}(\phi<0.01 ; r=0.988), \mathrm{AC}(\phi<0.01 ; r=0.970)$ and body mass index $(p<0.01 ; r=0.972)$.
}

Effect of calorie restricted diet

Received 22 January 2021 Revised 1 March 2021 Accepted 12 March 2021 
Originality/value - Low calorie diet has been proven to affect anthropometric development and has shown improvements in biochemical parameters of the rats. This may result in healthy aging which could prevent later-life diseases.

Keywords Nutrition, Obesity, Anthropometric, Biochemical, Calorie restricted diet

Paper type Research paper

\section{Introduction}

Obesity is a major public health concern worldwide because of its high prevalence within the past decade. According to WHO (2016) worldwide data, approximately more than 1.9 billion people of age $>18$ years are known to be overweight. Out of these, more than 650 million individuals were found to be obese (WHO, 2016). Obese or overweight is a serious condition which can lead to severe non-communicable diseases such as a cardiovascular disease or a kidney disease (Lambrinou et al., 2019). Moreover, obesity can give rise to agerelated health complications such as type 2 diabetes mellitus (T2DM), dyslipidemia, cancers and non-alcoholic fatty liver disease (Brahe et al., 2016). It is known to be one of the main risk factors contributing to motility and morbidity rate in chronic diseases (Szewczyk-Golec et al., 2017). Increased calorie intake remains to be one of the main causes giving rise to the obesity epidemic. For this reason, there is a growing demand for effective weight loss methods and diet strategies when it comes to treatment and prevention of obesity. Specifically, there is no effective long-term weight loss strategy proven safe to be used by overweight individuals. Energy restricted diets are currently the most popular and commonly used treatment in obesity (Dayan et al., 2019). However, this particular method of treatment greatly requires further studies to confirm their effects on biochemical and anthropometric parameters.

Previous studies have shown that a calorie intake reduction up to $20-50 \%$ without malnutrition can prolong lifespan up to $50 \%$ in some strains of rats and mice (Most et al., 2017). A chronic reduction of dietary energy intake (EI) by approximately $30 \%$ without incurrence of malnutrition has been found to be a broadly effective dietary intervention. It significantly decreases the adiposity, inflammation and is known to improve metabolic profiles among non-obese humans and rodents (O'Flanagan et al., 2017). Metabolic changes in response to lower caloric intake has proven to encourage certain safety characteristics such as improved insulin sensitivity and reduced blood glucose levels (Templeman et al., 2017). Moreover, it has shown to decrease chances of age-related complications such as cancer, diabetes, cardiovascular and neurodegenerative diseases in experimental models (O'Flanagan et al., 2017).

Restriction of calories without starvation is the most studied experimental technique among experimental models. Studies conducted in multiple animal models have shown that calorie restriction can improve health duration along with lifespan. Over the past two decades, the physiology of aging research community has held a close watch on calorie restriction studies on non-primates. In addition to these findings, there is plenty of continuous research ongoing to study the impact of calorie constrains on metabolic and molecular adaptations in humans (Most et al., 2017). Systematic reviews conducted in obese/ overweight women have showed that the amount of visceral adipose mass and inflammatory cytokine levels to have improved along with the adiponectin/leptin ratio upon consuming energy restricted diets (Razmpoosh et al., 2020). Furthermore, calorie restriction also remains to be the most successful intervention to date which has proven to delay the progression of aging and age-related chronic diseases. 
The study aims to determine the effect of calorie restricted diets on the anthropometric and biochemical parameters in Sprague Dawley (SD) rats. Thus, the effect of changes in body weight $(\mathrm{BW})$, body length (BL), abdominal circumference $(\mathrm{AC})$ and thoracic circumference $(\mathrm{TC})$ between high caloric intake (HCI), medium caloric intake (MCI) and low caloric intake (LCI) at different intervals were monitored. In addition to this, changes in biochemical parameters such as fasting blood glucose level (FGL), LDL, HDL and triglycerides (TG) cholesterol levels were also studied within the experimental groups. The study attempts to observe the consumption of food and EI between experimental intervals by all experimental animals.

Nutrition research and experimental studies often use animal models such as rodents as subjects instead of humans. Rodent animals such as rats and mice provide researchers with opportunities to study how factors such as genetics affect them. This can be achieved by using transgenic and knockout strains of mice (Vaughan et al., 2017). Moreover, animals are crucial for both ex vivo and in vivo nutrition studies as non-nutrient and nutrient elements present in food can alter with metabolic pathways by means of various unknown mechanisms. Hence, it is recommended to use animal models for nutrition-based studies before validating and translating it to humans (Chalvon-Demersay et al., 2017).

Some of the previous dietary intervention studies conducted in animals consisted of either monitoring their effects in relation to a certain drug or a specific disease. Up until now, most of the studies concerning anthropometry and biochemical parameters were conducted alongside physical activity. Therefore, current study was strictly focused on monitoring the effects of dietary intervention on anthropometric and biochemical parameters without inclusion of physical activity or any drug. Thus, this paper aims to determine the difference in anthropometric and biochemical parameters as an effect of consuming a calorie restricted diet by SD rats between experimental groups HCI, MCI and LCI. The results of this study showed important relationships between variables of anthropometric, biochemistry and nutritional parameters with regard to calorie restriction. Variations in EI per day have played a role in displaying differences between experimental groups.

\section{Methodology}

Research design

The experiment in this study used healthy SD rats obtained from Animal Laboratories Department Health of Science, Management and Sciences University (Shah Alam, Malaysia). This study used SD rats $(n=15)$ weighing $61.51 \pm 5.62 \mathrm{~g}$ (3 weeks old). They were each kept individually in standard animal housing at a controlled room temperature $\left(22.24^{\circ} \mathrm{C}\right)$ with 12 -h day/night cycle with free access to standard pellets, Gold Coin pallets (Port Klang, Malaysia) and tap water, ad libitum (Fattepur et al., 2018). The acclimatization period for the rats took 10 days after which all rats were weighed and divided into 3 experimental groups, namely, HCI $(n=5), \operatorname{MCI}(n=5)$ and LCI $(n=5)$. This study used the "resource equation" method for sample size calculation because of the lack of availability of effect size and standard deviation estimates from previous studies (Charan and Kantharia, 2013).

This study prepared three different diets. Calories for the diets were calculated in the form of energy per gram $(\mathrm{kJ} / \mathrm{g})$ before being fed to the respective experimental groups. For the purpose of growth requirements, the rats consumed 8-25 $\mathrm{g}$ amount of food intake gram per day (g/day) (Ritskes-Hoitinga and Chwalibog, 2002). This study implemented a standardized procedure for the groups. They were standardized by providing food consumption (FC) of $5 \mathrm{~g} / 100 \mathrm{~g}$ per BW of each rat (Atiqah et al., 2015).
Effect of calorie restricted diet 
The study fed the LCI group with only standard pellets and fed the HCI group with a homemade semi-solid and semi purified fat foods along with standard pellets (Hintze $e$ t al., 2018; Sheng et al., 2017). The study fed the MCI group $60 \%$ of the daily food intake of HCI diet (Hussain et al., 2016; Park et al., 2012). MCI group is considered as the control group in this study as their nutrient composition amounts are at a balance between HCI and LCI diet composition. LCI group is provided with a standard rat chow diet where in the fat component amounts up to $3 \%$ while the other nutrient components are restricted in comparison to $\mathrm{HCI}$ and MCI nutrient components. Hence is why, the LCI group is not considered as a control group in this study despite being fed a standard rat chow diet. The study used diet concepts prepared using diet dilution methods which are highlighted under diet preparation (Delorme et al., 1981; Nk and Naa, 2013; Samadi et al., 2017). For four weeks, the study monitored FC and the BW for careful characterization of energy ingestion and weight gain. In addition, the study assessed the anthropometric parameters and biochemical parameters on a weekly basis in all groups for four weeks.

\section{Ethical consideration}

This experiment was performed at the Animal House Laboratory, Management and Science University, Shah Alam, Malaysia. The Ethics Committee at Management and Science University approved all experimental protocols with respect to ethics code: MSU_RMC-02/ FR01/02/L3/020. All efforts were made to follow the ethical principles of animal handling and to minimize animal suffering.

\section{Diet preparation}

The HCI diet approximately contains $5.24 \mathrm{kcal} / \mathrm{g}$ with $8.4 \%$ protein, $19.5 \%$ carbohydrates, $61.2 \%$ fats and $10.9 \%$ others (Atiqah et al., 2015; Konopelnyuk et al., 2015). Ingredients used for the diet preparation were standard pellet ( $40 \mathrm{~g})$ and pure duck yolk fats ( $60 \mathrm{~g})$ (Echeverría et al., 2018; Getz and Reardon, 2006; Hintze et al., 2018; Karaçor et al., 2014). Diet preparations were carried out under sterile conditions on a weekly basis. The same ingredients were used in the preparation of MCI diet with $64 \mathrm{~g}$ of the standard pellet and $36 \mathrm{~g}$ of duck yolk fats. The LCI diet entirely consists of standard pellet. Every $100 \mathrm{~g}$ of a standard pellet diet contains $48.8 \%$ carbohydrates, $3 \%$ fats, $21 \%$ proteins and $27.2 \%$ others (Beheshti et al., 2017). Diet compositions and ingredient dilution followed for each diet were detailed in Tables 1 and 2, respectively.

\begin{tabular}{lccc}
\hline & & MCI & LCI \\
\hline Nutrients & $\% / 100 \mathrm{~g}$ & $\% / 100 \mathrm{~g}$ & $\% / 100 \mathrm{~g}$ \\
Carbohydrates & 19.5 & 31.2 & 48.8 \\
Protein & 8.4 & 13.4 & 21 \\
Fat & 61.2 & 37.9 & 3 \\
Others & 10.9 & 17.4 & 27.2 \\
Total energy & $5.24 \mathrm{kcal} / \mathrm{g}$ & $4.366 \mathrm{kcal} / \mathrm{g}$ & $3.062 \mathrm{kcal} / \mathrm{g}$
\end{tabular}

Table 1.

Diet composition for $100 \mathrm{~g}$ preparation
Notes: Other nutrients are made up of calcium, phosphorous, fiber, moisture and ash. A total of $60 \%$ of the $61.2 \%$ fats in HCI diet is from duck yolk fats. A total of $36 \%$ of $37.9 \%$ fats in MCI diet is from duck yolk fats 
The study used a measuring tape to measure the AC (immediately anterior to the forefoot), TC (immediately behind the foreleg) and BL (nose-anus length) of the rats (Konopelnyuk et al., 2015; Novelli et al., 2007). An electrical balance (A\&D GF3000) was used to monitor the BW. All the parameters were measured and the average for all parameters was calculated on a weekly basis for each group. BW and BL measurements were used to calculate the body mass index (BMI) and specific rate of body mass gain (SRBMG) of the rats as formulated below. The normal BMI for male rats is known to be between $0.45 \pm 0.02 \mathrm{~g} / \mathrm{cm}^{2}$ and $0.68 \pm 0.05 \mathrm{~g} / \mathrm{cm}^{2}$ (Atiqah et al., 2015):

- $\quad \mathrm{BMI}=\mathrm{BW}(\mathrm{g}) /$ length $^{2}\left(\mathrm{~cm}^{2}\right)$,

- $\operatorname{SRBMG}(\mathrm{g} / \mathrm{kg})=d M / \mathrm{Mdt}$,

where $d M$ is the gain of BW during $d t=t 2-t 1$ and $\mathrm{M}$ is the rat $\mathrm{BW}$ at $t 1$.

\section{Nutritional parameters}

Uneaten food was weighed everyday using an electrical balance. The uneaten food weight was then subtracted from the total amount of food provided to calculate the daily food intake. The food intake amount is considered to be the amount of food $(\mathrm{g})$ consumed by each rat within $24 \mathrm{~h}$ (Patel et al., 2017). The food intake amounts were used to calculate EI (kJ/day) and food efficiency rate (FER) (Novelli et al., 2007). The formulas used for the calculation are:

- $\quad \mathrm{EI}(\mathrm{kJ} / \mathrm{day})=$ mean of $\mathrm{FC} \times$ dietary metabolized energy.

- Food efficiency $(\mathrm{FE}, \mathrm{g}$ gained/kcal $)=($ mean of BW gain $\times 100) /$ total EI $(\mathrm{kcal})$.

\section{Biochemical parameters}

$\mathrm{SD}$ rats were fasted for $12 \mathrm{~h}$ or overnight for all weekly assessments of the biochemical parameters. The study used a $26 \mathrm{G}$ needle and a syringe to withdraw up to $0.2 \mathrm{ml}$ of blood from the marginal tail of the rats. The blood samples were collected during the fasting state

\begin{tabular}{|c|c|c|c|c|c|}
\hline $\mathrm{HCl}$ & & MCI & & LCI & \\
\hline Ingredients & Gram & Ingredients & Gram & Ingredients & Gram \\
\hline Standard pellet & 40 & Standard pellet & 64 & Standard pellet & 100 \\
\hline Duck yolk fat & 60 & Duck yolk fat & 36 & Duck yolk fat & 0 \\
\hline
\end{tabular}

Notes: *For $100 \mathrm{~g}$ of diet preparation, HCI diet consists of $40 \mathrm{~g}$ standard pellet with $60 \mathrm{~g}$ duck yolk fat. MCI diet is made up of $64 \mathrm{~g}$ standard pellet with $36 \mathrm{~g}$ of duck yolk fat. LCI is entirely $100 \mathrm{~g}$ standard pellet with no added extra ingredient

Table 2.

Diet dilution for $100 \mathrm{~g}$ preparation

\begin{tabular}{lcccc}
\hline EI $(\mathrm{kJ} / \mathrm{g})$ & Week 1 & Week 2 & Week 3 & Week 4 \\
\hline HCI & $3.29 \pm 0.01^{\mathrm{a}}$ & $6.02 \pm 0.01^{\mathrm{a}}$ & $7.95 \pm 0.01^{\mathrm{a}}$ & $9.71 \pm 0.01^{\mathrm{a}}$ \\
MCI & $2.18 \pm 0.01^{\mathrm{b}}$ & $3.99 \pm 0.00^{\mathrm{b}}$ & $5.28 \pm 0.01^{\mathrm{b}}$ & $6.45 \pm 0.00^{\mathrm{b}}$ \\
LCI & $1.08 \pm 0.00^{\mathrm{c}}$ & $1.98 \pm 0.00^{\mathrm{c}}$ & $2.62 \pm 0.00^{\mathrm{c}}$ & $3.20 \pm 0.00^{\mathrm{c}}$
\end{tabular}

Note: *EI between groups have increased in all 3 groups within the span of 4 weeks $\left({ }^{\mathrm{a}},{ }^{\mathrm{b}}\right.$ and ${ }^{\mathrm{c}}$ denote significant difference at $p<0.05$ between groups)

Table 3.

EI between groups for four weeks 
(Saxena et al., 2017). Fasting blood samples taken were used to monitor blood glucose levels as well as blood lipid profile. An Accu-check Oncall-plus glucose meter and test strips were used to determine FGL (Ghanbari et al., 2016). Readings were obtained in mg/dL. The blood lipid profiles of the rats were analyzed using a handheld 3-in-1 Combo Test Device for a complete lipid panel (Mission cholesterol meter). Blood samples collected for blood lipid profiling were allowed to stand for $15 \mathrm{~min}$ to clot. They were then centrifuged at 6,000 rev/ min for $5 \mathrm{~min}$. Up to $35 \mu 1$ of serum was collected with Pasteur pipette and introduced into cholesterol test strips. The results were displayed within $60 \mathrm{~s}$ after the insertion of the MEMO clip (Banda et al., 2018). HDL, TG and total cholesterol values obtained from the meter were used to calculate the LDL levels using the Fredwald's equation shown below (Francis et al., 2019).

$$
\text { LDL cholesterol }=\text { total cholesterol }-(\mathrm{HDL} \text { cholesterol })-(\mathrm{TG} / 5) \text {. }
$$

\section{Statistical analysis}

All data were analyzed using SPSS for windows version 23. The paper uses data reported as mean \pm standard deviation. Continuous variables with parametric distributions were analyzed using the analysis of variance (ANOVA) and if the results were significant, a posthoc test was performed. The differences between groups were defined to be statistically significant if $p$-value was less than 0.05 (Konopelnyuk et al., 2015). Differences of parameters between baseline and week four within groups were analyzed using repeated measures ANOVA.

\section{Results}

Food intake

All SD rats from week 1 to 4 had a FC of $8.48 \pm 0.24 \mathrm{~g}$ to $25.02 \pm 0.15 \mathrm{~g}$ of food. Based on the post-hoc results of week four, there was a significant difference in EI by rats at $p<0.05$ in three diet groups $(\mathrm{F}=3042872.02, p<0.0001)$. There was a significance difference in the FER of LCI at $p<0.05$ with MCI and HCI ( F = 59.55, $p<0.0001)$. As the FC and EI significantly increased up to four weeks, the rate of food efficiency significantly decreased among the rats as shown in Figure 1. Mean EI $(\mathrm{kJ} / \mathrm{g})$ for each experimental interval between groups are demonstrated in Table 1. According to Table 1, EI within groups have increased

Figure 1.

Changes in nutritional parameters between groups
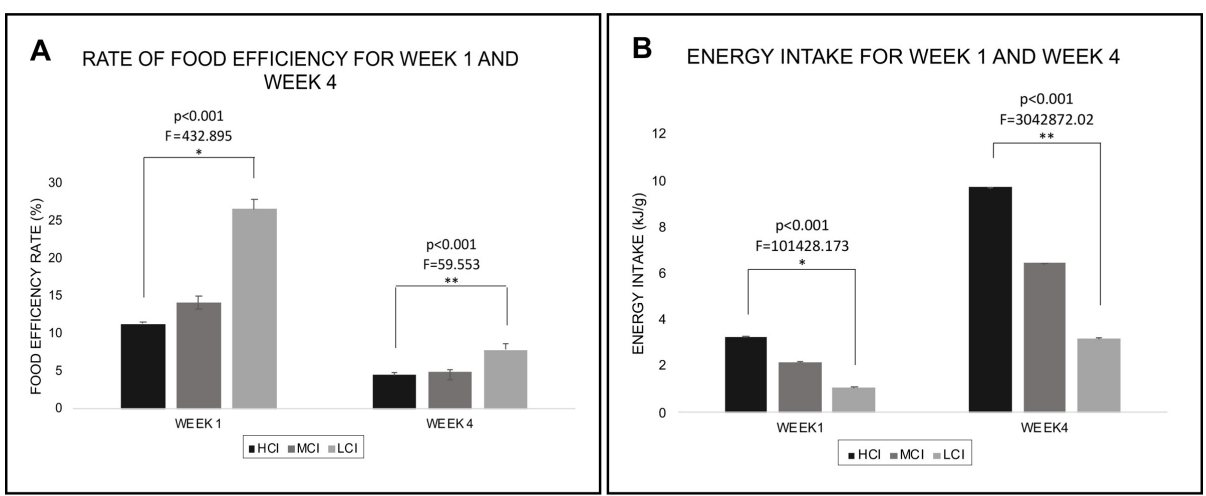

Notes: FER (\%) between groups at week one and week four (A). EI (kJ/g) between groups at week one and week four (B) 
significantly during the course of four weeks. EI for HCI, MCI and LCI increased from $3.29 \pm 0.01 \mathrm{~kJ} / \mathrm{g}$ to $9.71 \pm 0.01 \mathrm{~kJ} / \mathrm{g}, 2.18 \pm 0.01 \mathrm{~kJ} / \mathrm{g}$ to $6.45 \pm 0.01 \mathrm{~kJ} / \mathrm{g}$ and $1.08 \pm 0.01 \mathrm{~kJ} / \mathrm{g}$ to $3.20 \pm 0.01 \mathrm{~kJ} / \mathrm{g}$, respectively, at $p<0.0001$. Increase is observed to be highest in HCI group followed by MCI and LCI groups.

Analysis of anthropometric parameters. BW significantly increased in all SD rats by week four between HCI (mean, MCI and LCI experimental groups). The mean score for BW significantly increased in $\mathrm{HCI}(\mathrm{M}=230.44, \mathrm{SD}=1.47), \mathrm{MCI}(\mathrm{M}=204.30, \mathrm{SD}=0.72)$ and LCI $(\mathrm{M}=188.53, \mathrm{SD}=1.50)$ at $p<0.05$. Significant increase was observed in average $\mathrm{BL}$ at week 4 in $\mathrm{HCI}(\mathrm{M}=19.12$, $\mathrm{SD}=0.08)$, $\mathrm{MCI}(\mathrm{M}=18.84$, $\mathrm{SD}=0.05)$ and $\mathrm{LCI}(\mathrm{M}=18.54$, $\mathrm{SD}=0.05)$. The average weekly increase of BW and BL for all experimental groups is shown in Table 4. The weekly mean BMI and abdominal thoracic circumference (ACTC) ratio between groups are displayed in Table 5. Figure 2A shows the weekly increase of BMI. A significant effect was observed in BMI of the rats at $p<0.05$ in all three groups $(\mathrm{F}=316.27, p<0.0001)$. The SRBMG gain was significantly decreased with the increase of the age of the SD rats used (Figure 2B). AC (Figure 2C) and TC (Figure 2D) have increased throughout the experiment.

There was a significant difference in TC at $p<0.05$ in LCI $(\mathrm{M}=10.38, \mathrm{SD}=0.12)$ with both $\mathrm{MCI}(\mathrm{M}=10.50, \mathrm{SD}=0.12)$ and $\mathrm{HCI}(\mathrm{M}=10.72, \mathrm{SD}=0.08)$. Significant increase was recorded in the ACTC ratio (Figure $2 \mathrm{E})$ between $\mathrm{LCI}(\mathrm{M}=1.02, \mathrm{SD}=0.01)$ and $\mathrm{HCI}(\mathrm{M}=$ $1.04, \mathrm{SD}=0.01)$ at $p=0.002$. Based on the Pearson correlation results, a strong positive correlation was observed between the final BW and the final BL of the SD rats at week $4, r=$ $0.957, n=15$ and $p<0.0001$.

Analysis of biochemical parameters. According to FGL demonstrated in the Figure 3A, levels remained the same for $\mathrm{HCI}$ group at week one and week four. A decrease is observed in FGL at week four among the LCI group in comparison to week one levels. However, there was no significant difference in FGL at $p<0.05$ in LCI $(\mathrm{M}=87.93$, SD $=1.50)$ with both MCI $(\mathrm{M}=88.65, \mathrm{SD}=1.50)$ and $\mathrm{HCI}(\mathrm{M}=90.09, \mathrm{SD}=1.27)$ at week four. Repeated measured ANOVA conducted between HCI and LCI group at week one and week four showed no significant difference in FGL ( $p=0.585$ and $p=0.091$, respectively).

Figure $3 \mathrm{~B}$ shows that LDL levels remained constant between groups at week $1 . \mathrm{LDL}$ levels for HCI group and MCI group have increased from week one to week four while the levels remained the same for the LCI group. Increase appears to be highest among the HCI group in comparison to MCI group. There is a significant difference in LDL cholesterol level observed at week four for HCI group $(\mathrm{M}=18.44, \mathrm{SD}=1.61 \mathrm{mg} / \mathrm{dL})$ than with the MCI group $(\mathrm{M}=13.80, \mathrm{SD}=0.37 \mathrm{mg} / \mathrm{dL})$ and LCI group $(\mathrm{M}=11.28, \mathrm{SD}=0.30 \mathrm{mg} / \mathrm{dL})$ at $p<0.05$. Repeated measured ANOVA conducted to compare LDL levels between HCI and LCI group at week one and week four showed a significant difference at week four $(\phi<0.001)$.

Figure 3D shows the HDL levels among experimental groups at week one and week four. Week one of the experiment demonstrates a constant HDL level between the HCI (M $=68.40$, $\mathrm{SD}=1.14), \mathrm{MCI}(\mathrm{M}=69.40, \mathrm{SD}=0.54)$ and $\mathrm{LCI}(\mathrm{M}=69.20, \mathrm{SD}=0.83)$ groups. A decrease in HDL levels is observed in both HCI and MCI groups at week four while the HDL level remained at a constant for $\mathrm{LCI}$. There is a significant difference noticed in week-four HDL levels between $\mathrm{HCI}(\mathrm{M}=49.40, \mathrm{SD}=0.54 \mathrm{mg} / \mathrm{dL})$ with $\mathrm{MCI}(\mathrm{M}=59.40, \mathrm{SD}=0.54 \mathrm{mg} / \mathrm{dL})$ and $\mathrm{LCI}(\mathrm{M}=68.60, \mathrm{SD}=0.0 .54 \mathrm{mg} / \mathrm{dL})$ at $p<0.0001$.

TG levels between groups for week one and week four are shown in the Figure 3C. TG levels at week four for HCI, MCI and LCI were noted to be (M = 142.80, SD = 1.92 mg/dL), $(\mathrm{M}=$ 106.20, $\mathrm{SD}=1.64 \mathrm{mg} / \mathrm{dL})$ and $(\mathrm{M}=94.40, \mathrm{SD}=0.54 \mathrm{mg} / \mathrm{dL})$, respectively. No significant increase is observed for LCI group from week one $(\mathrm{M}=92.40, \mathrm{SD}=1.67)$ to week four $(\mathrm{M}=$ $94.40, \mathrm{SD}=0.54)$. An increase in TG levels is observed for both HCI and MCI group from week
Effect of calorie restricted diet 
NFS

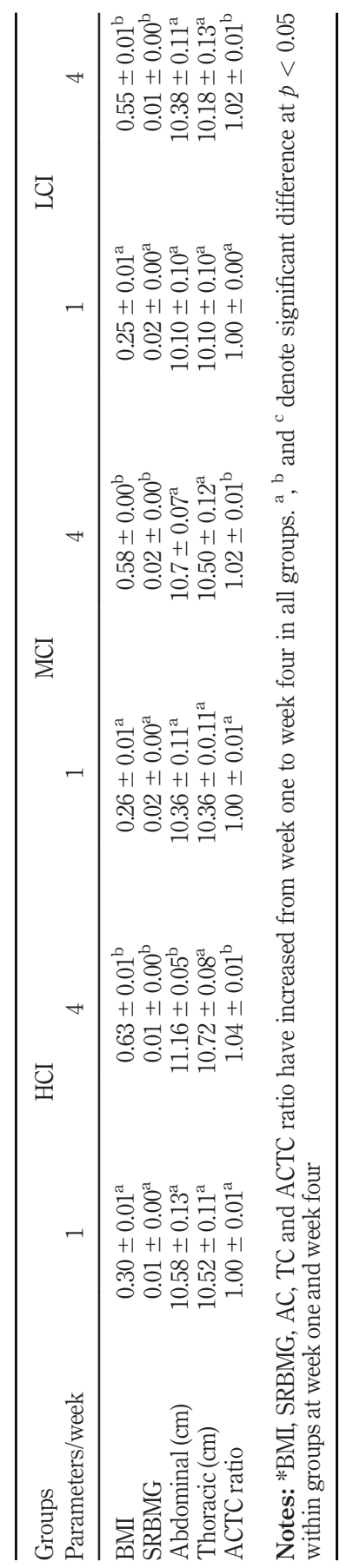

Table 4.

BMI $(\mathrm{g} / \mathrm{cm} 2)$,

SRBMG $(\mathrm{g} / \mathrm{kg}), \mathrm{AC}$ (cm), TC $(\mathrm{cm})$ and ACTC ratio at week one and week four

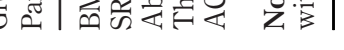


one to week four. According to the post-hoc results of week four, there is a significant increase of TG levels for both HCI group and MCI group at $p<0.0001$.

\section{Discussion}

The study used repeated measures ANOVA to study the difference in anthropometric and biochemical parameters within groups during the beginning (baseline) and end (week four) of the experiment. It helped to understand and assess the changes taking place within the groups over time. Present study shows a significant increase in BW, BMI and ATCT ratio between groups at week four. The HCI group was found to show a greater increase in these parameters in comparison to MCI and LCI. This increase is because of increased EI via a high calorie diet. Amount of EI and FER among groups showed an increase from week one to week four (Figure 1). Although the LCI group rats did not show any decrease in these parameters, the rate of increase in these parameters was noticeably lesser than the other two experimental groups. According to Romieu et al. (2017), increased energy consumption is the major cause behind excessive weight gain leading to obesity (Romieu et al., 2017). Obesity is a condition highly correlating to oxidative stress. The condition can cause free radicals in the body to increase while simultaneously reducing the number of antioxidants neutralizing them. This could lead to chronic inflammation and adverse effect into cell survival within the body. A study conducted by Sandra et al. (2019) demonstrated the suppression of high fat diet (HFD)-induced liver steatosis by co-administrating the antioxidant hydroxytyrosol (HT) and $n$-3 PUFA docosahexaenoic acid (DHA) in weaning male mice. The study used 14 animals. They were randomly divided into control diet group and HFD group. Each group was supplemented with either saline or HT or DHA or HT and DHA. The experiment results showed an increase in liver fat contents such as FFA, TGs and cholesterol for the HFD group. HFD group further showed a significant correlation with SREBP-1c mRNA levels. SREBP-1c upregulation in the liver can activate expression of other genes in the liver involved in FA synthesis such as PERK, ATF6 $\alpha$, ACC, FAS and SCoAD-1. These changes were paralleled by a down regulation of PPAR $\alpha$ in HFD. PPAR $\alpha$ down regulation can cause oxidative stress enhancement in the liver. This was evidenced by the increased ROS and TBARS (Soto-Alarcón et al., 2019). The oxidative stress effect is further supported by the study conducted by Takatsu et al. (2013). They used DahlS.Z-Lepr ${ }^{f a} / \operatorname{Lepr}^{f a}$ (DS/obese) rats which belong to a cross breed between Zucker rats and Dahl-salt sensitive. The rats were divided into a normal group consuming a chow diet and a $65 \%$ calorie restricted diet. The experiment was done for a period of 13 weeks. Findings of the results showed a reduction in ameliorated left ventricular hypertrophy, body fat content, fibrosis and diastolic dysfunction among DS/obese rats present in calorie restricted group. Moreover, results further showed calorie restriction to have attenuated the inflammation in the rats as well as oxidative stress.

\begin{tabular}{lccrcrc}
\hline & \multicolumn{3}{c}{ BW (g) } \\
Week & HCI & MCI & LCI & HCI & MCI & LCI \\
\hline 0 & $67.92 \pm 1.62^{\mathrm{a}}$ & $60.52 \pm 2.53^{\mathrm{b}}$ & $56.08 \pm 3.45^{\mathrm{b}}$ & $18.50 \pm 0.00^{\mathrm{a}}$ & $18.62 \pm 0.04^{\mathrm{b}}$ & $18.52 \pm 0.04^{\mathrm{a}}$ \\
1 & $104.96 \pm 1.24^{\mathrm{a}}$ & $91.59 \pm 2.8^{\mathrm{b}}$ & $85.03 \pm 4.72^{\mathrm{c}}$ & $18.66 \pm 0.05^{\mathrm{a}}$ & $18.62 \pm 0.04^{\mathrm{a}}$ & $18.52 \pm 0.04^{\mathrm{b}}$ \\
2 & $151.20 \pm 0.73^{\mathrm{a}}$ & $130.73 \pm 3.19^{\mathrm{b}}$ & $122.27 \pm 5.85^{\mathrm{c}}$ & $18.76 \pm 0.05^{\mathrm{a}}$ & $18.62 \pm 0.04^{\mathrm{b}}$ & $18.52 \pm 0.04^{\mathrm{c}}$ \\
3 & $186.66 \pm 3.88^{\mathrm{a}}$ & $172.59 \pm 1.46^{\mathrm{b}}$ & $163.52 \pm 1.18^{\mathrm{c}}$ & $18.82 \pm 0.04^{\mathrm{a}}$ & $18.64 \pm 0.05^{\mathrm{b}}$ & $18.62 \pm 0.04^{\mathrm{b}}$ \\
4 & $230.4 \pm 1.47^{\mathrm{a}}$ & $204.30 \pm 0.722^{\mathrm{b}}$ & $188.54 \pm 1.50^{\mathrm{c}}$ & $19.12 \pm 0.08^{\mathrm{a}}$ & $18.84 \pm 0.05^{\mathrm{b}}$ & $18.54 \pm 0.05^{\mathrm{c}}$
\end{tabular}

Notes: *Overall increase in BW and BL are observed in all groups for four weeks. ${ }^{\mathrm{a}},{ }^{\mathrm{b}}$ and ${ }^{\mathrm{c}}$ denote significant difference at $p<0.05$ between groups

Effect of calorie restricted diet

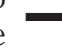


NFS

Figure 2.

Anthropometric changes between groups

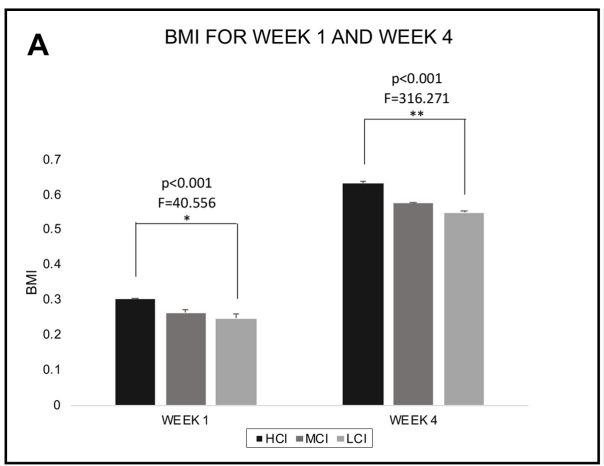

B SPECIFIC RATE OF BODY MASS GAIN FOR WEEK
AND WEEK 4

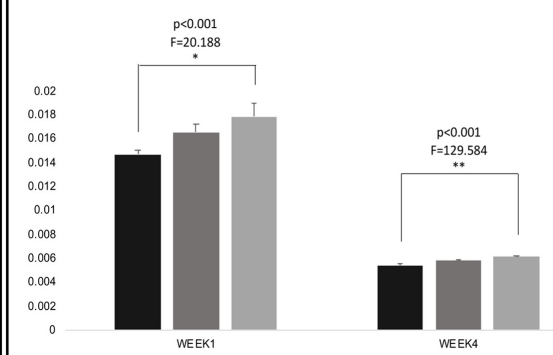

WEEK1

$-\mathrm{HCl}=\mathrm{MCl}=\mathrm{LCl}$

C ABDOMINAL CIRCUMFERENCE FOR WEEK 1 AND

D THORACIC CIRCUMFERENCE FOR WEEK 1 AND
WEEK 4
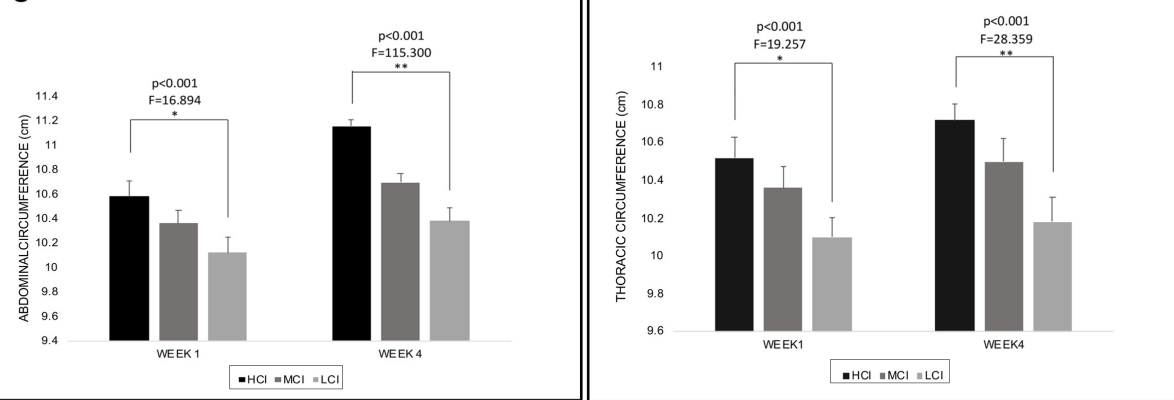

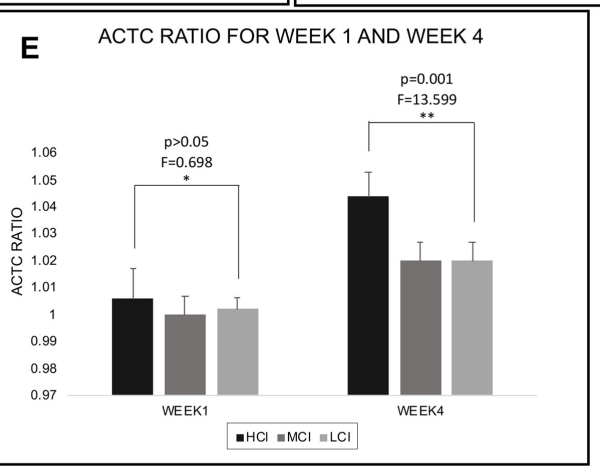

Notes: BMI between groups at week one and week four (A). SRBMG between groups at week one and week four (B). AC between groups at week one and week four (C). TC between groups at week one and week four (D). ACTC ratio between groups at week one and week four (E)

Apart from this, the expression of angiotensin II type $1 \mathrm{~A}$ receptor genes and the angiotensin-converting enzyme present in the heart were found to be dysregulated (Takatsu et al., 2013). Oxidative stress is a process known to be linked with age-related and aging diseases such as cancer, neurodegeneration, diabetes and cardiovascular diseases. Calorie restriction is a regimen known to prevent age-related diseases by reducing oxidative stress. Experimental studies have shown that calorie restriction can reduce aging by decreasing the 

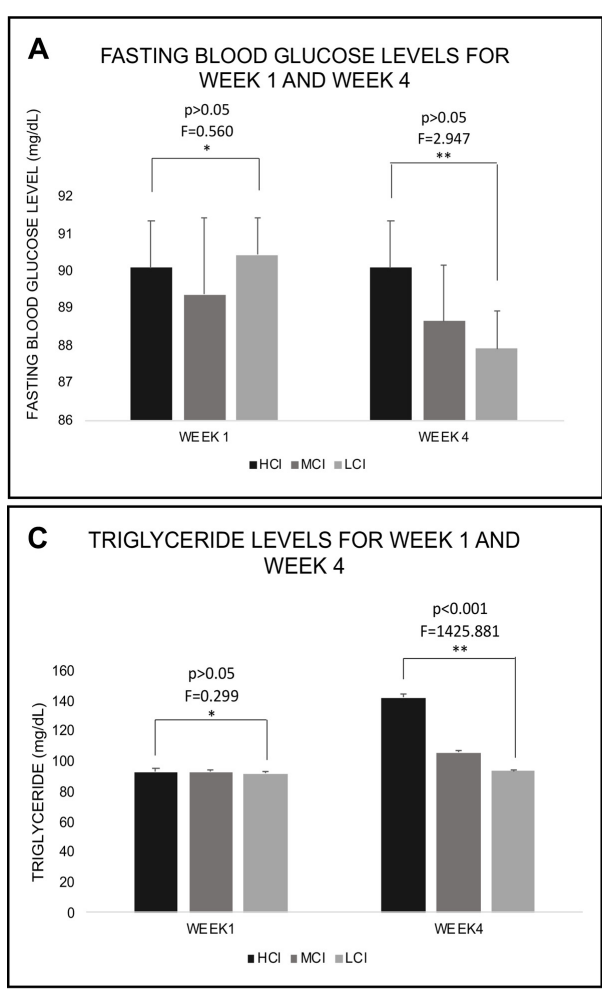

\section{D}

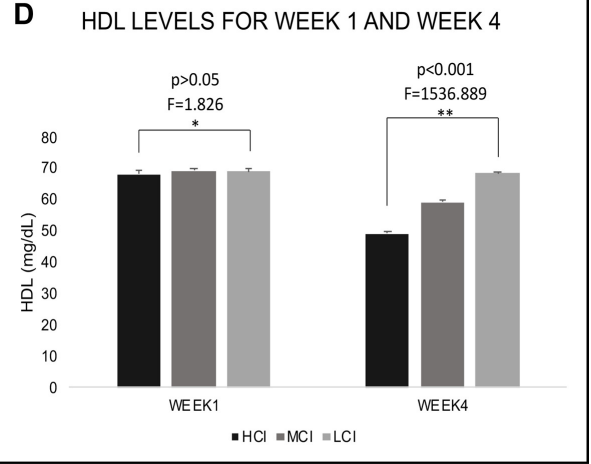

Notes: FGL (mg/dL) between groups at week zero and week four (A). LDL levels (mg/dL) between groups at week zero and week four (B). TG levels $(\mathrm{mg} / \mathrm{dL})$ between groups at week zero and week four (C). HDL levels (mg/dL) between groups at week zero and week four (D)
Effect of calorie restricted diet

Figure 3. Changes in biochemical parameters between groups

amount of ROS production in mitochondria and increasing the antioxidant enzyme activity. Antioxidant enzymes such as the catalase, glutathione and superoxide dismutase are responsible for detoxifying ROS and other harmful radicals (Walsh et al., 2014). A study conducted by Daniele La et al. (2020) found that calorie restriction can reverse aging in aged and obese rats. The study used young and aged rats where aged rats where divided into two groups (control group and calorie restriction group). Calorie restriction was given for a period of six months. The results showed that calorie restriction increased the plasma adiponectin levels and simultaneously reduced the levels of anti-inflammatory protein TSG6 pro-inflammatory marker. It brought about improvements in redox balance along with inflammatory process in tissues and plasma levels. This was supported by decreased expression of GSTP-1 anti-oxidant. GSTP-1 is an anti-oxidant which possess proliferative activity (La Russa et al., 2020). Based on these studies, we can safely agree that calorie restriction can promote healthy aging in rodents.

HCI group rats were recorded to have a significantly high amount of LDL cholesterol levels. Eating a high calorie diet can cause LDL levels to increase. This in turn increases TG levels in the blood and causes a reduction in HDL cholesterol levels. HCI rats were recorded to have low HDL levels and high TG levels in this study. Increased EI could lead to an 
accumulation of fat in the body. The findings of this study are in line with a study conducted by Gao et al. (2017) to study the effect of dietary restriction (DR) in hyperlipidemic mice. The study was conducted for a period of five weeks. Mouse models were divided into three groups, namely, HFD, DR 30\% group and DR 50\% group. DR 30\% group consumed $70 \%$ of HFD while DR $50 \%$ consumed $50 \%$ of HFD. The results of the study showed a significant decrease in serum lipids along with serum glucose levels, liver index, average BW and liver weight among both DR groups in oppose to HFD (Gao et al., 2017). A different study done by Salesi and Mehrtash (2018) examined the effects of calorie restricted diet on lipid coat proteins of SD rats. Lipid coat proteins are proteins which can prevent lipolysis of adipose tissue. A total of 30 male adult SD rats were used in this study. After eight weeks of acclimatization, the rats were divided into two groups: calorie restricted diet group and HFD group. PCR was used to assess the expression levels of lipid coat proteins while HOMAindex was used to calculate the insulin resistance. Findings of this study reported to have a significant decrease of weight and insulin resistance in calorie restricted group. It also showed that expression levels of lipid coat protein perilupin-1 were higher in the skeletal muscle of the HFD group in comparison to calorie restricted group. Thus, this study demonstrates that calorie restriction can prevent insulin resistance along with the accumulation of fat (Salesi and Mehrtash, 2018). Additionally, our data is further supported by a study done by Kim et al. (2016) where they investigated the metabolic changes in obesity induced SD rats. The study used a Hyperpolarized ${ }^{13} \mathrm{C}$ magnetic resonance spectroscopy to measure the metabolic and serum enzyme levels. Ten SD rats of seven weeks old were randomly divided into two groups. Five rats were fed a HFD while remaining five rats were fed a normal diet. Serum biochemistry results showed HFD rats to have significantly increased cholesterol and serum ALT levels. Increased levels of metabolites such as alanine pyruvate ratio were observed in HFD rats along with increased BW and LDH levels. Macro vesicular steatosis and ballooning degeneration in hepatocytes were identified in histopathological examination of liver tissues of HFD rats (Kim et al., 2016).

Several animal research studies have shown that calorie restriction boosts cardiovascular function by reducing cholesterol levels of LDL and TG (Longo et al., 2015). Calorie restriction has been known to reverse the effects of obesity-related cardiovascular diseases. This is caused as a result of increased HDL levels. HDL concentration is a traditional marker used as risk stratification for atherosclerotic cardiovascular diseases. HDL possesses cardio protective properties because of its anti-oxidant and anti-diabetic properties. They have the potential to inhibit vascular inflammation and enhance endothelial function (Barter and Genest, 2019). Calorie restriction can ameliorate the two major risk factors of an ischemic heart disease known as hyperglycemia and hyperlipidemia (Fontana and Klein, 2007). Furthermore, it has been known to reduce the formation of atherosclerotic lesion along with the accumulation of visceral fat (Guo et al., 2002). In the present experiment, LCI group rats were found to have reduced levels of LDL cholesterol and TG from week one to week four of the study (Figure 3B and C). It was also noticed that LCI rats had increased HDL cholesterol in week four when compared to week one (Figure 3D). These results are in line with the experiment conducted by Melo et al. (2016). Their study investigated the effects of calorie restriction on risk factors of cardiovascular diseases in adult Wistar rats. The results showed that calorie restricted Wistar rats had increased cardiac functions with reduced lipid profiles for LDL and TG (Melo et al., 2016). Apart from the aforementioned study, an experiment conducted by Ciobanu et al. (2017) tested the hypothesis that calorie restriction can reduce the incidence of age-related metabolic syndrome and obesity. This strategy is thought to have potential for preventing consequences relating to an ischemic stroke in the future. SD rats used for this experiment were randomly 
divided into 3 groups: 30 young aged control, 30 aged control and 30 aged calorie restricted group. The experiment was conducted for a period of eight weeks. The rats were fasted overnight and then anesthetized to carry out the experimental infarction. At the end of the study, it was found that there was a continuous reduction in BW among aged control rats. Whereas, no significant BW decrease was observed in those rats which consumed the calorie restricted diet. A significant post-stroke increase was seen in serum glucose, IGF-1 levels and insulin among calorie restricted group rats. This means that there is a possibility for enhancement in recovery when a rat already consuming a calorie restricted diet is subjected to stroke. At the same time, the study's findings showed that over nutrition can lead to slow recovery from stroke (Ciobanu et al., 2017). Hence, with regard to this literature, care should be given to avoid over nutrition or over feeding to laboratory-based animals as it is considered unhealthy.

Apart from obesity, increased calorie intake can potentially cause increased adipose store. This could lead to other chronic disorders such as metabolic syndrome and T2DM (Nikhra, 2018). Metabolic syndrome consists of multiple combinations of metabolic disorders such as dyslipidemia, hyperlipidemia, obesity and impaired glucose tolerance. In the current study, LCI rats showed a decrease in FGL throughout the duration of this experiment while it remained more or less the same for HCI and MCI rats (Figure 3A). Increased blood glucose level can be caused because of T2DM as a result of insulin resistance. Intake of high calorie diet could result a spike in blood glucose levels. However, in case of LCI without malnutrition, the blood glucose amount can be decreased to an appropriable amount in blood thereby preventing the risk of T2DM. A study conducted by Wong et al. (2018) have shown that intaking a high carbohydrate high fat $(\mathrm{HCHF})$ diet can result in significant changes in metabolic syndrome parameters. This experiment was conducted for 16 weeks. A total of 14 male Wistar rats were used in this experiment. At the beginning of the study, they were randomly divided into two groups: HCHF diet and normal standard chow diet group. Results showed a significant increase in systolic blood pressure, LDL cholesterol, dyslipidemia and glucose intolerance among HCHF rats in comparison to normal rats, thereby indicating that metabolic syndrome can be induced among rats consuming a HCHF diet (Wong et al., 2018). In addition to this, Park et al. (2006) conducted a study by using 1.5 months and 18 months old rats to investigate the effect of calorie restriction in young age and middle age rats. The rats were subjected to $30 \%$ calorie restriction for a period of four to six weeks. Immunoblotting for protein abundance of phosphorylated insulin receptors was used for the analysis of quadriceps femoris muscle. They observed that calorie restriction greatly improved glucose tolerance and showed lower serum insulin response to glucose in both younger and middle age rats (Park et al., 2006).

\section{Limitations}

The study was conducted for a period of one month. This is a considerably short duration of time to study the effects and changes of calorie restriction effectively. As a result of this, the current study was unable to report the long-term effects of consuming a calorie restricted diet. The sample size of the rats used in this experiment is less. Moreover, the rats used for this experiment are younger in terms of age and are not obese. This limits the extent to which accurate conclusions can be drawn when doing anti-aging and nutrition studies for obesity prevention. Zucker rats are known to be a good animal model for obesity studies. However, this experiment uses SD rats as the animal model. Therefore, additional correlation studies using obese Zucker rats are greatly needed to further confirm anti-aging effects of DR. Furthermore, only two biochemical parameters (blood glucose and blood lipid profile) were focused on this experiment, which is insufficient to confirm the long-term
Effect of calorie restricted diet 
effects of calorie restriction on biochemical aspects. This study further lacked histological evidences which are crucial to evaluate the extent of damage HCI can do to tissues.

\section{Conclusion}

In conclusion, our caloric restriction experiment showed significant changes in anthropometric measurements such as BMI, AC, TC and ACTC ratio and biochemical markers such as blood lipid profiles and blood glucose levels in SD rats. The nutrition parameters of EI and FER displayed how they could affect anthropometric and biochemical parameters based on the number of calories consumed by the diet. This study shows that efficiency of calorie intake and FC can affect blood cholesterol levels and BMI significantly. In future thought, more calorie restricted studies involving longer experimental duration is essential to support the findings of this study. In addition to this, other laboratory methods such as histological analysis and more molecular level analysis are required to gain a better understanding of the mechanism and morphological changes taking place inside the body as a result of consuming a calorie restricted diet. More experimental studies are needed to study the advanced effects of calorie restriction on parameters such as insulin level, growth hormones and organ weight before translating the results to humans. More repetition studies involving the aforementioned parameters could pave a way for an effective diet plan to be used in the treatment or prevention of obesity in the future.

\section{References}

Atiqah, N., Abdul, A., Rahmat, A. and Jaafar, H.Z.E. (2015), Protective Effects of Tamarillo (Cyphomandra Betacea) Extract against High Fat Diet Induced Obesity in Sprague-Dawley Rats. 2015.

Banda, M., Nyirenda, J., Muzandu, K. and Sijumbila, G. (2018), Antihyperglycemic and Antihyperlipidemic Effects of Aqueous Extracts of Lannea Edulis in Alloxan-Induced Diabetic Rats, Vol. 9, pp. 1-8, doi: 10.3389/fphar.2018.01099.

Barter, P. and Genest, J. (2019), "HDL cholesterol and ASCVD risk stratification: a debate", Atherosclerosis, Vol. 283, pp. 7-12, doi: 10.1016/j.atherosclerosis.2019.01.001.

Beheshti, R., Treesukosol, Y., Igusa, T. and Moran, T.H. (2017), A Predictive Model of Rats 'Calorie Intake as a Function of Diet Energy Density.

Brahe, L.K., Astrup, A. and Larsen, L.H. (2016), "Can we prevent obesity-related metabolic diseases by dietary modulation of the gut microbiota?", Advances in Nutrition, Vol. 7 No. 1, pp. 90-101, doi: 10.3945/an.115.010587.

Chalvon-Demersay, T., Blachier, F., Tomé, D. and Blais, A. (2017), "Animal models for the study of the relationships between diet and obesity: a focus on dietary protein and estrogen deficiency", Frontiers in Nutrition, Vol. 4, p. 1, doi: 10.3389/fnut.2017.00005.

Charan, J. and Kantharia, N. (2013), "How to calculate sample size in animal studies?", Journal of Pharmacology and Pharmacotherapeutics, Vol. 4 No. 4, pp. 303-306, doi: 10.4103/0976500X.119726.

Ciobanu, O., Elena Sandu, R., Tudor Balseanu, A., Zavaleanu, A., Gresita, A., Petcu, E.B., ... PopaWagner, A. (2017), "Caloric restriction stabilizes body weight and accelerates behavioral recovery in aged rats after focal ischemia”, Aging Cell, Vol. 16 No. 6, pp. 1394-1403, doi: 10.1111/ acel.12678.

Dayan, P.H., Sforzo, G., Boisseau, N., Pereira-Lancha, L.O. and Lancha, A.H. (2019), "A new clinical perspective: treating obesity with nutritional coaching versus energy-restricted diets", Nutrition, Vol. 60, pp. 147-151, doi: 10.1016/j.nut.2018.09.027. 
Delorme, C.B., Wojcik, J. and Gordon, C. (1981), "Method of addition of cellulose to experimental diets and its effect on rat growth and protein utilization", The Journal of Nutrition, Vol. 111 No. 9, pp. 1522-1527, doi: 10.1093/jn/111.9.1522.

Echeverría, F., Valenzuela, R., Bustamante, A., Álvarez, D., Ortiz, M., Soto-Alarcon, S.A., . . Videla, L. A. (2018), "Attenuation of high-fat diet-induced rat liver oxidative stress and steatosis by combined hydroxytyrosol-(HT-) eicosapentaenoic acid supplementation mainly relies on HT", Oxidative Medicine and Cellular Longevity, Vol. 2018, doi: 10.1155/2018/5109503.

Fattepur, S., Nilugal, K.C., Rajendran, R., Asmani, F. and Yusuf, E. (2018), “Anti-hyperlipidemic activity of methanolic extract of boesenbergia pandurata (finger root) in experimental induced hypercholestrolemic Sprague Dawley rats", Asian Journal of Pharmaceutical and Clinical Research, Vol. 11 No. 15, pp. 8-12, doi: 10.22159/ajpcr.2018.v11s3.29962.

Fontana, L. and Klein, S. (2007), “Aging, adiposity, and calorie restriction”, JAMA, Vol. 297 No. 9, pp. 986-994, doi: 10.1001/jama.297.9.986.

Francis, D., Ani, C., Nworgu, C., Pamela, O., Uzoma, I., Uzoigwe, J., . . Daniel, N. (2019), “The effect of ethanolic seed extract of citrullus lanatus (watermelon) on blood glucose level and lipid profile of diabetic Wistar rats", European Journal of Medicinal Plants, Vol. 26 No. 4, pp. 1-6.

Gao, H.T, Cheng, W.Z, Xu, Q. and Shao, L.X. (2017), "Dietary restriction reduces blood lipids and ameliorates liver function of mice with hyperlipidemia", Journal of Huazhong University of Science and Technology [Medical Sciences], Vol. 37 No. 1, pp. 79-86, doi: 10.1007/s11596-0171698-8.

Getz, G.S. and Reardon, C.A. (2006), "Diet and murine atherosclerosis", Arteriosclerosis, Thrombosis, and Vascular Biology, Vol. 26 No. 2, pp. 242-249, doi: 10.1161/01.ATV.0000201071.49029.17.

Ghanbari, E., Sc, M., Nejati, V., Ph, D., Khazaei, M. and Ph, D. (2016), "Improvement in serum biochemical alterations and oxidative stress of liver and pancreas following use of royal jelly in streptozotocin-induced diabetic rats", Cell Journal (Yakhteh), Vol. 18 No. 3, pp. 362-370.

Guo, Z.M., Mitchell-Raymundo, F., Yang, H., Ikeno, Y., Nelson, J., Diaz, V., ... Reddick, R. (2002), "Dietary restriction reduces atherosclerosis and oxidative stress in the aorta of apolipoprotein Edeficient mice”, Mechanisms of Ageing and Development, Vol. 123 No. 8, pp. 1121-1131, doi: 10.1016/S0047-6374(02)00008-8.

Hintze, K.J., Benninghoff, A.D., Cho, C.E. and EWard, R. (2018), "Modeling the Western diet for preclinical investigations", Advances in Nutrition, Vol. 9 No. 3, pp. 263-271, doi: 10.1093/ advances/nmy002.

Hussain, M.A., Abogresha, N.M., Hassan, R., Tamany, D.A. and Lotfy, M. (2016), "Effect of feeding a high-fat diet independently of caloric intake on reproductive function in diet-induced obese female rats”, Archives of Medical Science, Vol. 4 No. 4, pp. 906-914, doi: 10.5114/aoms.2016.59790.

Karaçor, K., Çam, M., Orhan, N., Coşgun, E. and Demirin, H. (2014), "High fatty diet effects on rat liver", Electronic Journal of General Medicine, Vol. 11 No. 2, pp. 99-108, doi: 10.15197/sabad.1.11.47.

Kim, G.W., Ahn, K.Y., Kim, Y.H. and Jeong, G.W. (2016), "Time-course metabolic changes in high-fat diet-induced obesity rats: a pilot study using hyperpolarized 13C dynamic MRS”, Magnetic Resonance Imaging, Vol. 34 No. 8, pp. 1199-1205, doi: 10.1016/j.mri.2016.06.002.

Konopelnyuk, V., Kot, L., Makai, S. and Ostapchenko, L. (2015), "Effect of trigonella Foenum-Graecum seed powder on anthropometrical and nutritional parameter", Journal of Applied Pharmaceutical Science, Vol. 5 No. 11, pp. 95-100, doi: 10.7324/JAPS.2015.501116.

La Russa, D., Marrone, A., Mandalà, M., Macirella, R. and Pellegrino, D. (2020), “Antioxidant/antiInflammatory effects of caloric restriction in an aged and obese rat model: the role of adiponectin", Biomedicines, Vol. 8 No. 12, p. 532, doi: 10.3390/biomedicines8120532.

Lambrinou, E., Hansen, T. and Beulens, J. (2019), "Lifestyle factors, self-management and patient empowerment in diabetes care”, European Journal of Preventive Cardiology, Vol. 26 No. 2_suppl, pp. 55-63, doi: 10.1177/2047487319885455. 
Longo, V.D., Antebi, A., Bartke, A., Barzilai, N., Brown-Borg, H.M., Caruso, C., .. . Fontana, L. (2015), "Interventions to slow aging in humans: are we ready?", Aging Cell, Vol. 14 No. 4, pp. 497-510, doi: $10.1111 /$ acel.12338.

Melo, D.S., Costa-Pereira, L.V., Santos, C.S., Mendes, B.F., Costa, K.B., Santos, C.F.F., . . Dias-Peixoto, M.F. (2016), "Severe calorie restriction reduces cardiometabolic risk factors and protects rat hearts from ischemia/reperfusion injury", Frontiers in Physiology, Vol. 7 No. APR, p. 106, doi: 10.3389/fphys.2016.00106.

Most, J., Tosti, V., Redman, L.M. and Fontana, L. (2017), "Calorie restriction in humans: an update", Ageing Research Reviews, Vol. 39, pp. 36-45, doi: 10.1016/j.arr.2016.08.005.

Nikhra, V. (2018), "Cardiovascular disease, obesity, MetS, T2DM and aging, and benefits of calorie restriction and calorie restriction mimetics heart and aging view project diabetes and aging view project", available at: www.researchgate.net/publication/325654759.

Nk, S. and Naa, B. (2013), "Two experiments were carried out to compare two techniques (amino lysine is an essential amino acid closely associated with body protein", Revista Brasileira de Ciencia Avicola, Vol. 15 No. 2, pp. 123-134, doi: 10.1590/S1516-635X2013000200008.

Novelli, E.L.B., Diniz, Y.S., Galhardi, C.M., Ebaid, G.M.X., Rodrigues, H.G., Mani, F., . . Filho, J.L.V.B.N. (2007), "Anthropometrical parameters and markers of obesity in rats", Laboratory Animals, Vol. 41 No. 1, pp. 111-119.

O'Flanagan, C.H., Smith, L.A., McDonell, S.B. and Hursting, S.D. (2017), "When less may be more: Calorie restriction and response to cancer therapy", BMC Medicine, Vol. 15 No. 1, doi: 10.1186/ s12916-017-0873-x.

Park, S., Komatsu, T., Hayashi, H., Yamaza, H., Chiba, T., Higami, Y., . . Shimokawa, I. (2006), “Calorie restriction initiated at middle age improved glucose tolerance without affecting age-related impairments of insulin signaling in rat skeletal muscle", Experimental Gerontology, Vol. 41 No. 9, pp. 837-845, doi: 10.1016/j.exger.2006.06.055.

Park, S., Park, N., Valacchi, G. and Lim, Y. (2012), "Calorie restriction with a High-Fat diet effectively attenuated inflammatory response and oxidative stress-related markers in obese tissues of the high diet fed rats", Mediators of Inflammation, Vol. 2012, doi: 10.1155/2012/984643.

Patel, A.R., Patra, F., Shah, N.P. and Shukla, D. (2017), "Biological control of mycotoxins by probiotic lactic acid bacteria", Dynamism in Dairy Industry and Consumer Demands, Vol. 2015 No. February, pp. 2-4, doi: 10.1155/2015.

Razmpoosh, E., Zare, S., Fallahzadeh, H., Safi, S. and Nadjarzadeh, A. (2020), "Effect of a low energy diet, containing a high protein, probiotic condensed yogurt, on biochemical and anthropometric measurements among women with overweight/obesity: a randomised controlled trial", Clinical Nutrition ESPEN, Vol. 35, pp. 194-200, doi: 10.1016/j.clnesp.2019.10.001.

Ritskes-Hoitinga, M. and Chwalibog, A. (2002), "Nutrient requirements, experimental design, and feeding schedules in animal experimentation. Handbook of laboratory animal science", Essential Principles and Practices, 2nd ed., Vol. 1, pp. 281-310, doi: 10.1201/b10416-12.

Romieu, I., Dossus, L., Barquera, S., Blottière, H.M., Franks, P.W., Gunter, M., .. W Willett, W.C. (2017), "Energy balance and obesity: what are the main drivers?", Cancer Causes and Control, Vol. 28 No. 3, pp. 247-258, doi: 10.1007/s10552-017-0869-z.

Salesi, M. and Mehrtash, M. (2018), "The role of caloric restriction on lipid coat proteins gene expression and insulin resistance after 8 weeks high caloric diet in male Rats - Journal of arak university of medical sciences", Retrieved July 14, 2020, available at: http://jams.arakmu.ac.ir/browse.php? a_id $=5604 \&$ slc_lang $=$ en\&sid $=1 \&$ printcase $=1 \&$ hbnr $=1 \& \mathrm{hmb}=1$

Samadi, Wecke, C., Pastor, A. and Liebert, F. (2017), "Assessing lysine requirement of growing chicken by direct comparison between supplementation technique and "Gottingen approach", Open Journal of Animal Sciences, Vol. 07 No. 1, pp. 56-69, doi: 10.4236/ojas.2017.71006.

Saxena, A.K., Al-Ani, I. and Talib, N.A. (2017), Anti-Atherosclerotic Effects of Eurycoma Longifolia (Tongkat Ali) in Rats Fed on High-Fat Diet, (June). 
Sheng, H., Ha, S., Chew, S., Phang, W., Ban, J., Tan, L. and Abdul, K. (2017), "Increased susceptibility of post-weaning rats on high-fat diet to metabolic syndrome", Journal of Advanced Research, Vol. 8 No. 6, pp. 743-752, doi: 10.1016/j.jare.2017.10.002.

Soto-Alarcón, S.A., Ortiz, M., Orellana, P., Echeverría, F., Bustamante, A., Espinosa, A., [. . .] Videla, L. A. (2019), "Docosahexaenoic acid and hydroxytyrosol co-administration fully prevents liver steatosis and related parameters in mice subjected to high-fat diet: a molecular approach", BioFactors, Vol. 45 No. 6, pp. 930-943, doi: 10.1002/biof.1556.

Szewczyk-Golec, K., Rajewski, P., Gackowski, M., Mila-Kierzenkowska, C., Wesołowski, R., Sutkowy, P., ... Woźniak, A. (2017), "Melatonin supplementation lowers oxidative stress and regulates adipokines in obese patients on a calorie-restricted diet", Oxidative Medicine and Cellular Longevity, Vol. 2017, pp. 8494107, doi: 10.1155/2017/8494107.

Takatsu, M., Nakashima, C., Takahashi, K., Murase, T., Hattori, T., Ito, H., ... Nagata, K. (2013), "Calorie restriction attenuates cardiac remodeling and diastolic dysfunction in a rat model of metabolic syndrome", Hypertension, Vol. 62 No. 5, pp. 957-965, doi: 10.1161/HYPERTENSIONAHA.113.02093.

Templeman, N.M., Flibotte, S., Chik, J.H.L., Sinha, S., Lim, G.E., Foster, L.J., .. Johnson, J.D. (2017), "Reduced circulating insulin enhances insulin sensitivity in old mice and extends lifespan", Cell Reports, Vol. 20 No. 2, pp. 451-463, doi: 10.1016/j.celrep.2017.06.048.

Vaughan, K.L., Kaiser, T., Peaden, R., Anson, R., Cabo, R. and Mattison, J. (2017), "Caloric restriction study design limitations in rodent and nonhuman primate studies", The Journals of Gerontology: Series A, doi: 10.1093/gerona/glx131.

Walsh, M.E., Shi, Y. and Van Remmen, H. (2014), "The effects of dietary restriction on oxidative stress in rodents", Free Radical Biology and Medicine, Vol. 66, pp. 88-99, doi: 10.1016/j.freeradbiomed.2013.05.037.

WHO (2016), "Obesity and overweight”, Retrieved August 25, 2020, available at: www.who.int/newsroom/fact-sheets/detail/obesity-and-overweight.

Wong, S.K., Chin, K.Y., Suhaimi, F.H., Ahmad, F. and Ima-Nirwana, S. (2018), “The effects of a modified high-carbohydrate high-fat diet on metabolic syndrome parameters in male rats", Experimental and Clinical Endocrinology and Diabetes, Vol. 126 No. 4, pp. 205-212, doi: 10.1055/s-0043-119352.

\section{Further reading}

Subar, A.F., Freedman, L.S., Tooze, J.A., Kirkpatrick, S.I., Boushey, C., Neuhouser, M.L., .. KrebsSmith, S.M. (2015), "Addressing current criticism regarding the value of self-report dietary data", The Journal of Nutrition, Vol. 145 No. 12, pp. 2639-2645, doi: 10.3945/jn.115.219634.

\section{Corresponding author}

Zulhabri Othman can be contacted at: zulhabri_othman@yahoo.com

For instructions on how to order reprints of this article, please visit our website: 